\title{
All nonadherence is equal but is some more equal than others? Tuberculosis in the digital era
}

\author{
Helen R. Stagg $\mathbb{0}^{1}$, Mary Flook ${ }^{1}$, Antal Martinecz ${ }^{2,3,4}$, Karina Kielmann ${ }^{5}$, \\ Pia Abel Zur Wiesch (10),3,13, Aaron S. Karat ${ }^{5,6,13}$, Marc C.I. Lipman (10,8,13, \\ Derek J. Sloan $\mathbb{1}^{9,13}$, Elizabeth F. Walker ${ }^{10}$ and Katherine L. Fielding (1) ${ }^{11,12}$
}

Affiliations: 'Usher Institute, University of Edinburgh, Edinburgh, UK. ${ }^{2}$ Department of Biology, Pennsylvania State University, University Park, PA, USA. ${ }^{3}$ Center for Infectious Disease Dynamics, Huck Institutes of the Life Sciences, Pennsylvania State University, University Park, PA, USA. ${ }^{4}$ Department of Pharmacy, Faculty of Health Sciences, UiT - The Arctic University of Norway, Tromsø, Norway. ${ }^{5}$ The Institute for Global Health and Development, Queen Margaret University, Musselburgh, UK. ${ }^{6}$ TB Centre, London School of Hygiene \& Tropical Medicine, London, UK. "UCL Respiratory, Division of Medicine, University College London, London, UK. ${ }^{8}$ Department of Respiratory Medicine, Royal Free London NHS Foundation Trust, London, UK. ${ }^{9}$ School of Medicine, University of St Andrews, St Andrews, UK. ${ }^{10}$ London School of Hygiene \& Tropical Medicine, London, UK. ${ }^{11}$ Department of Infectious Disease Epidemiology, London School of Hygiene \& Tropical Medicine, London, UK. ${ }^{12}$ School of Public Health, University of the Witwatersrand, Johannesburg, South Africa. ${ }^{13}$ These authors contributed equally.

Correspondence: Helen R. Stagg, Usher Institute, University of Edinburgh, Old Medical School, Teviot Place, Edinburgh, EH8 9AG, UK. E-mail: helen.staggded.ac.uk

ABSTRACT Adherence to treatment for tuberculosis (TB) has been a concern for many decades, resulting in the World Health Organization's recommendation of the direct observation of treatment in the 1990s. Recent advances in digital adherence technologies (DATs) have renewed discussion on how to best address nonadherence, as well as offering important information on dose-by-dose adherence patterns and their variability between countries and settings. Previous studies have largely focussed on percentage thresholds to delineate sufficient adherence, but this is misleading and limited, given the complex and dynamic nature of adherence over the treatment course. Instead, we apply a standardised taxonomy - as adopted by the international adherence community - to dose-by-dose medication-taking data, which divides missed doses into 1) late/noninitiation (starting treatment later than expected/not starting), 2) discontinuation (ending treatment early), and 3) suboptimal implementation (intermittent missed doses). Using this taxonomy, we can consider the implications of different forms of nonadherence for intervention and regimen design. For example, can treatment regimens be adapted to increase the "forgiveness" of common patterns of suboptimal implementation to protect against treatment failure and the development of drug resistance? Is it reasonable to treat all missed doses of treatment as equally problematic and equally common when deploying DATs? Can DAT data be used to indicate the patients that need enhanced levels of support during their treatment course? Critically, we pinpoint key areas where knowledge regarding treatment adherence is sparse and impeding scientific progress.

@ERSpublications

Digital adherence technologies (DATs) provide a wealth of information on dose-by-dose anti-TB medication-taking. Studies of DAT data should place nonadherence in standardised taxonomic frameworks in order to best inform intervention and regimen design. https://bit.ly/3jq1D8a

Cite this article as: Stagg HR, Flook M, Martinecz A, et al. All nonadherence is equal but is some more equal than others? Tuberculosis in the digital era. ERJ Open Res 2020; 6: 00315-2020 [https:// doi.org/10.1183/23120541.00315-2020]. 


\section{Introduction}

Many decades after trials of antimicrobials for tuberculosis (TB) [1], the standard treatment for drug-sensitive disease remains lengthy at 6 months; regimens for drug-resistant disease can last for 2 years [2]. Concerns about adherence to treatment over such long periods - and the implications of that nonadherence - led to the World Health Organization (WHO) recommendation of directly observed treatment (DOT) in 1994 $[3,4]$.

In recent years, digital adherence technologies (DATs; including SMS-based reminders, video-supported therapy [VOT], and medication monitor boxes) have increasingly been tested as remote alternatives to DOT/other standards of care as they are potentially cheaper, more acceptable, and less financially and temporally burdensome $[5,6]$. DATs provide healthcare workers with regular, up-to-date, information on how medication has been taken (either accessed at each appointment or remotely each day). DATs can be provided in different ways, e.g. to all patients as the sole source of support or as part of a package of interventions that is personalised to an individual's needs [7]. Intervention packages may be reviewed as a result of appointment-by-appointment (or remote dose-by-dose) evaluation of DAT data that demonstrates the need for enhanced treatment support $[8,9]$. Such reviews could also help to determine the patients least in need of dose-by-dose monitoring, i.e. providing a "step-down" approach during treatment.

Like DOT, DATs are interventions to promote dose-taking that assumes all doses are of equal importance. This one-size-fits-all approach latently assumes that missed doses are essentially interchangeable, i.e. that each is of equal importance in terms of its clinical implications. This may not be the case; early-stage adherence when bacterial loads are higher may be more important than late stage, for example. Additionally, it is assumed that DOT and DATs work equally well across the entire treatment period, which is not always true [10].

The advent of DATs provides a unique moment to reassess our global approach to nonadherence to anti-TB medications. Assuming that DAT event monitoring is equivalent to dose-taking, DAT devices provide rich digital datasets of date- and time-stamped information that have not previously been available to the research community. Key lessons about anti-TB medication-taking and best practice for DAT deployment can be learnt, in order to avoid a simple duplication of our current global DOT approach and better personalise clinical care.

In this paper, we take the opportunity of ongoing global evaluation and roll-out of DATs to review and refine a classification of nonadherence to treatment, examine the evidence for the global burden and association of different types of nonadherence with treatment outcomes, and consider what this refined classification of nonadherence means for intervention and regimen design and deployment.

\section{What is nonadherence?}

In this paper, we adopt a definition of adherence that emphasises the patient's role in agreeing a treatment plan i.e.:

Adherence - when a patient's dose-taking, at any stage during treatment, matches mutually agreed recommendations from the prescriber [11].

Therefore, nonadherence represents a divergence from this agreement.

Traditionally, TB research has assessed nonadherence using simple 80-90\% thresholds of doses taken across the duration of treatment. To date, few studies have determined whether $80-90 \%$ is the optimal point of inflection. Furthermore, this simple binary classification masks extensive complexity across the treatment period. Given this complexity, it is essential to lay out definitions and descriptors [12, 13]. In 2010, partly coordinated by the European Society for Patient Adherence, Compliance and Persistence (ESPACOMP), a new taxonomy for nonadherence was launched [14]. This is the only globally accepted taxonomy for nonadherence, which consists of three core concepts, mappable using dose-by-dose data, such as that provided by DATs (figure $1 \mathrm{a}$ and $\mathrm{b}$ ):

1) Initiation, which tracks when the first dose of a regimen is taken relative to the intended start date.

2) Discontinuation, which documents the cessation of treatment.

3) Implementation - how doses are taken during the period of persistence (the time frame between initiation and discontinuation), i.e. intermittent missed doses (treatment gaps).

As a condition with a time-limited treatment period, TB lends itself to this definitions framework. Drug-sensitive TB is treated for 6 months with an all-oral regimen, starting with four drugs administered over 2 months (initiation phase; not to be confused with treatment initiation), followed by two drugs over 4 months (continuation phase) [15]. It is usually dosed daily; in some places, thrice-weekly regimens (although problematic in their own right, see below) are utilised to allow to make DOT less burdensome 
a)

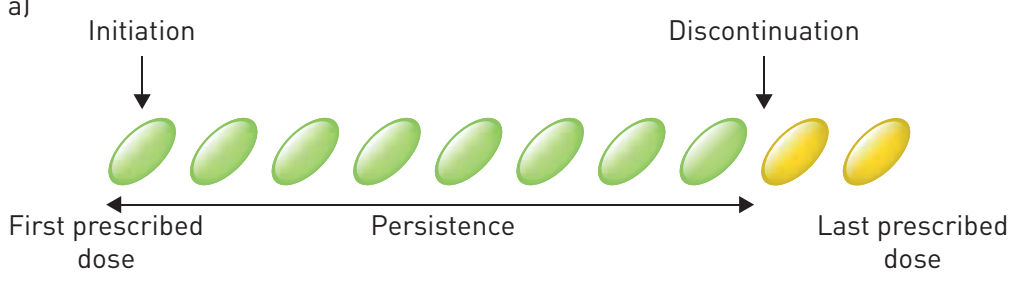

b)

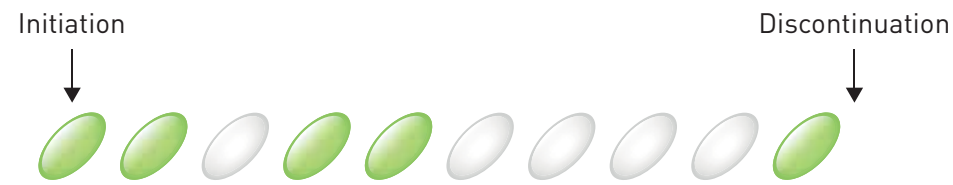

First prescribed

Implementation

Last prescribed dose

dose

c)

Patient
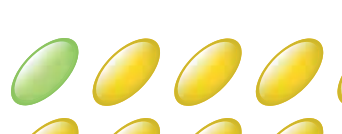

Doses taken

1

2
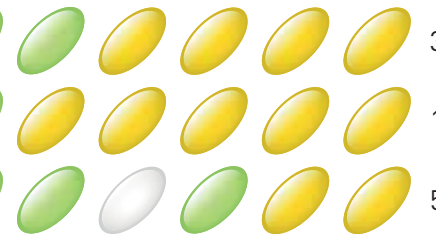

$33 \%$

3
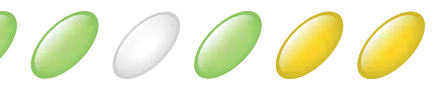

$17 \%$

4
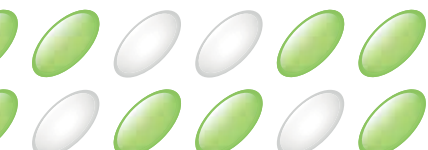

5
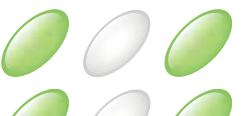

o.

$67 \%$

Doses missed $22 / 48=46 \%$

Individuals discontinuing early $3 / 8=38 \%$

$67 \%$ Dose missed due to discontinuation $11 / 22=50 \%$

6

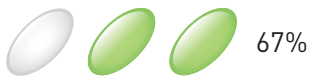

7

$(2$

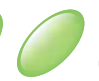

10
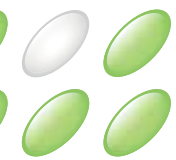

$67 \%$

8

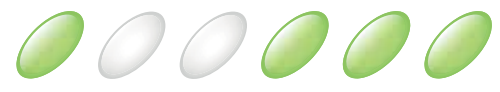

$67 \%$

d)

\section{Patient}

1
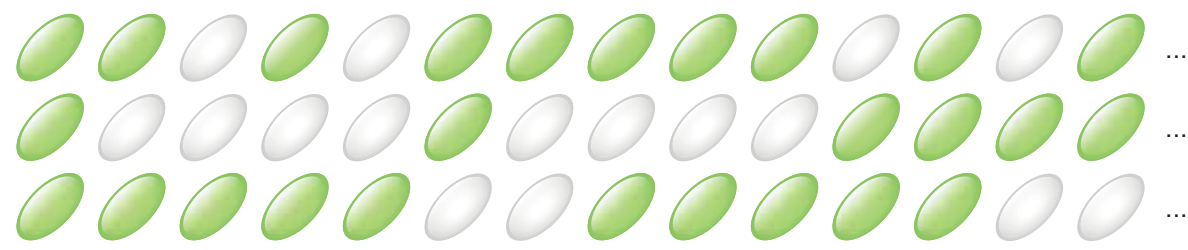

FIGURE 1 The different components of nonadherence to treatment. Using the standard taxonomy described by VRIJENS et al. [14], it is possible to distinguish between the first and last prescribed doses of medication and the first and last doses taken. In terms of sources of nonadherence: firstly (panel (a)), individuals may initiate treatment later than agreed with their clinician. Secondly, treatment may be discontinued early, i.e. before the last prescribed dose. Persistence is the period between initiation and discontinuation. Thirdly (panel (b)), nonadherence arises from how individuals implement their medication; doses may be missed intermittently. In this diagram, the complete regimen is only 10 doses. Panel (c) shows the impact of discontinuation within an illustrated population of eight patients taking six doses of treatment each before treatment is stopped. 38\% (1-3) discontinue their treatment early, all at different time points. $75 \%$ of patients (3-8) display some form of suboptimal implementation. Despite this, doses missed due to discontinuation make up half of nonadherence across the entire patient population. Panel (d) illustrates different types of suboptimal implementation. Patient 1 - short, irregular gaps. Patient 2 - long, irregular gaps. Patient 3 - regular gaps. Treatment is not stopped after the last illustrated dose. Green - dose taken, white - missed due to suboptimal implementation, orange - missed due to discontinuation. Panels (a) and (b) adapted from VRIJENS et al. [14]. 
on both the patient and the healthcare system. Fixed dose combination (FDC) pills are used in many settings. Thus the number of treatment doses expected to be taken in a week can vary from place to place and patient to patient; the number of pills this represents will also vary depending upon a patient's weight. For drug-resistant TB, both regimens themselves and their dosing becomes more complex, and treatment lengthier [16].

Within the context of nonadherence to TB treatment, the core concepts can be mapped as follows:

1) Late initiation of, or not initiating, treatment: this charts the time frame between the intended treatment start date after a patient is informed of their diagnosis and the first dose being taken. The reasons for issues with initiation are multifactorial. Delays can be due to a lag in, or nonacquisition of, medication, as well as provided medication not being taken. Noninitiation may be driven by failures in the access of/ linkage to care cascade with drivers and consequences that are, therefore, different from late initiation.

2) Early discontinuation of treatment, e.g. due to loss to follow-up (LFU; previously known as default) [17].

3) Suboptimal implementation [12], i.e. the form of nonadherence that has been the focus of both observational studies and clinical trials.

Of note, LFU - as defined by the WHO [17] - is not a clear-cut proxy for early discontinuation of treatment. This is because it is both a standardised end-of-treatment outcome that is reported within surveillance systems (treatment is interrupted for two consecutive months or more), as well as occurring when a TB patient does not start treatment ("initial LFU” or "pretreatment LFU") [18, 19]. LFU thus 1) contains some noninitiation and 2) is not the only form of discontinuation due to the time constriction placed upon it. Furthermore, LFU documents nonengagement with clinical appointments, not medication-taking per se.

In the following sections, we discuss how the effect of the three core concepts of nonadherence on TB control depends on two factors: 1) the prevalence of each kind of nonadherence and 2) the impact of each type on treatment outcomes. Throughout this paper, we use a previously published dataset of DAT data to provide a worked example of the concepts that we illustrate (table 1).

\section{Late or noninitiation}

What is the global burden?

Among our core components of nonadherence, noninitiation is arguably most on the global map, as a component part of the WHO's campaign to find and treat the "missing millions" [21]. The precise number of patients not starting treatment is unknown, although WHO estimates treatment coverage to be $69 \%$

\section{TABLE 1 The implications of nonadherence patterns for intervention and regimen design: worked example from China}

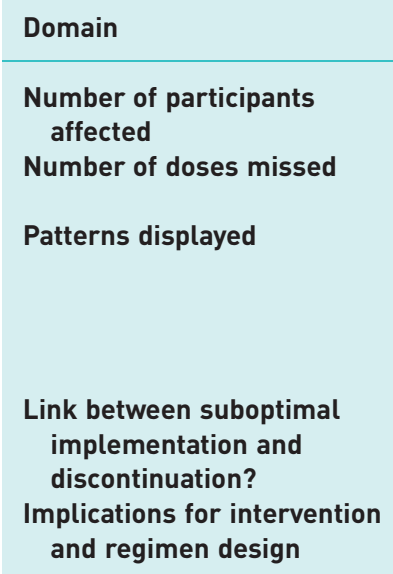

\section{Domain}

Suboptimal implementation

$748 / 780(95.9 \%)$ of all participants suboptimally implemented their treatment.

$9487 / 16794(56.4 \%)$ missed doses were due to suboptimal implementation.

The median gap length per patient was one dose, with a maximum number of gaps per participant of 24. $176 / 780$ individuals $(22.6 \%)$ had gaps of seven doses (a fortnight) or more. Suboptimal implementation increased over time.

Missed doses in the initiation phase due to suboptimal implementation associated with increased risk of discontinuation in the continuation phase.

The causes of large numbers of short gaps need to be ascertained and addressed by an effective intervention.

\section{Discontinuation}

$235 / 780(30.1 \%)$ of all participants discontinued early.

$7307 / 16794$ (43.5\%) missed doses were due to early discontinuation.

$5.1 \%$ of individuals had discontinued treatment by the end of month $2,14.4 \%$ by the end of month 4 $18.2 \%$ by the end of month $5,36.3 \%$ by the end of month 6 lincluding individuals missing only their last dose).

Given the burden of discontinuation and when it occurs, shortened regimens may have been helpful in this setting. Early-stage suboptimal implementation could act as an indicator of patients who require an intervention to prevent discontinuation

In a study of 780 patients from a pragmatic cluster-randomised trial in China of electronic reminders to improve treatment adherence [9, 12], data were taken from the control arm of the trial (electronic reminders set to silent, thus no intervention to promote adherence). Medication monitor boxes provided granular data as to whether each individual dose was taken (box opening used as a proxy). Treatment was dosed every other day. All patients initiated treatment within this study. Decision-making as to which type of nonadherence should be targeted by interventions will also depend upon the relative impact of each form of nonadherence on outcomes [20]. 


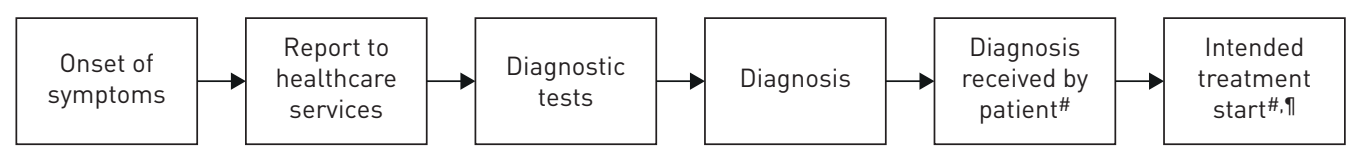

FIGURE 2 Cascade of care until the start of TB treatment. \#: These two time points may be on the same day. ": For drug-resistant TB patients, drug sensitivity testing results may not be available until after treatment for drug-sensitive disease is initiated, necessitating a change in regimen.

globally [22]. For rifampicin-resistant TB, BoyD et al. [23] have estimated a similar global mean of $76 \%$ of individuals initiating treatment, among those diagnosed. In a review of studies undertaken in low-income and lower-middle-income countries, or those with a high burden of TB, MacPherson et al. [18] projected that $18 \%$ of individuals do not initiate treatment after diagnosis in African nations and $13 \%$ in Asian nations. A later study estimated the figure to be $12 \%$ in South Africa [19].

A series of systematic reviews and meta-analyses have examined temporal delays in treatment initiation (the time frame between diagnosis and the start of treatment). In India among pulmonary TB patients, median delay was 2.5 days (IQR 1.9-3.6) [24] and in the Eastern Mediterranean Region 0 to 2 days [25]. In comparison, a recent observational study from China found the median time from TB diagnosis to multidrug-resistant (MDR)-TB treatment was 6 months [26]. This is because the situation in drug-resistant disease is even more complex, because patients may start on the 6-month regimen whilst waiting for drug sensitivity testing results before their treatment is adjusted (proving a window for further drug resistance to develop) [16], and sourcing second-line drugs may take time.

What is the relationship with treatment outcomes?

Examining the relationship between initiation of treatment and treatment outcomes is complicated by the different measures of lateness used in the literature. In many papers, an overall figure of the delay between symptoms and the start of treatment was quoted, rather than delays between diagnosis and the start of treatment (figure 2). In sensu stricto, we sought to document delays between diagnosis (preferably when it was received by the patient) and the start of treatment only.

In a 2018 review, MELSEw et al. [27] examined the impact of delays in starting treatment on patient infectiousness. Among eight studies, four found evidence for an association between delays in treatment initiation after the onset of symptoms (with a roughly doubling of the odds of infectiousness), three found no evidence for an association, and one found mixed evidence. The delays charted were from less than a fortnight to more than 90 days.

Evidence from Ethiopia documented a doubling in the adjusted relative risk of treatment failure, death or LFU among those for whom delay was $>30$ days [28]. This study used a measure of "overall delay" (from the start of symptoms to the start of treatment) with a median of 55 days (interquartile range [IQR] 32-100) documented. Of this, 22 days (9-48) were classed as "provider delay", i.e. the time post-presentation at a healthcare facility between diagnosis and the start of treatment.

Among MDR patients in Myanmar, in a univariable analysis where treatment delay was classified as between the date of MDR-TB confirmation and the date of treatment initiation, the median treatment delay for patients with poor treatment outcomes (lost to follow-up, failed, died) was 144 days, which was longer than among patients who achieved successful treatment outcomes (102 days) [29]. In an adjusted analysis comparing the impact of long ( $\geqslant$ median of 152 days) versus short $(<$ median) delays, this association was not retained.

In MDR-TB patients in China results were also mixed, this time depending upon the measure of delay used. The time between TB diagnosis and the start of MDR treatment showed some effect, albeit with a null-inclusive confidence interval, whereas shorter delays ( $\leqslant 60$ days) after the performance of drug sensitivity testing showed a doubling or more in the likelihood of a positive treatment outcome, depending upon the other factors adjusted for [26].

\section{Discontinuation}

What is the global burden?

Due to the substantial overlap with LFU and the use of this measure as a standardised reporting outcome, the estimates of the global burden of discontinuation have been captured in many studies. An individual patient data meta-analysis of 9000 MDR-TB patients from 23 countries suggested around a sixth were lost to follow-up, with a median timing of 7 months [30]. In an older systematic review not specifically for drug-resistant disease, KRUK et al. [31] documented likelihoods of LFU of 7-54\% and timings of between 42 and 85 days in low- and middle-income settings. There were large amounts of variation between 
countries and regions. Approaches that include a precise analysis of when discontinuation from treatment occurs and how this relates to LFU should become more common as dose-by-dose monitoring systems are rolled out globally.

\section{What is the relationship with treatment outcomes?}

Determining the relationship between discontinuation and treatment outcomes is complex, given the use of LFU both as a marker of discontinuation and a negative surveillance outcome [17]. Useful sources of data include the randomised controlled trials (RCTs) that developed the standard regimen we use today. Historically, it was the addition of rifampicin and then pyrazinamide which allowed treatment to be shortened to 6 months [32]; further studies showed an important increase in the likelihood of post-treatment relapse when treatment was reduced to 4 months [33].

In recent years, several RCTs have sought to shorten the treatment of drug-sensitive TB to 4 months by including fluoroquinolones but, as yet, none have demonstrated noninferiority [34-36]. Pooled analyses have indicated that such regimens may be noninferior in particular patient groups, indicating the need for stratified treatment approaches [37]. Although such regimens are intended to reduce nonadherence by shortening overall duration, this may increase the sensitivity of such regimens to suboptimal implementation, i.e. the importance of each dose in the regimen may be increased, relative to a longer regimen, making each missed dose more problematic.

Critically, well-designed studies using dose-by-dose monitoring systems such as DATs, together with robust treatment outcome collection, will go a long way towards answering remaining questions in this area.

\section{Suboptimal implementation}

What is the global burden?

Until recently, suboptimal implementation for anti-TB treatment has been assessed through the differentially reliable self-reported or questionnaire-derived methods (for example $[38,39]$ ) and DOT (e.g. $[10,40-42])$. Study protocols also used various thresholds to classify nonadherence, and often reported a mixture of suboptimal implementation and discontinuation in their analyses.

To date, the burden of suboptimal implementation is suggested to be highly variable between countries and regions, e.g. $21.3 \%$ in a pooled estimate from Ethiopia versus $90.8 \%$ in the Philippines, although differences will partly be protocol-dependent $[42,43]$. Approaches that include a precise analysis of the types of suboptimal implementation displayed by patients should become more common as dose-by-dose monitoring systems are rolled out globally [44], e.g. examining the lengths of gaps displayed and when they occur during treatment $[12,45]$. For example, in a recent study in China, $47.2 \%$ of 780 patients had a dosing gap of a week or more and $95.9 \%$ some form of suboptimal implementation (table 1) [12].

\section{What is the relationship with treatment outcomes?}

There has been substantial interest in the relationship between suboptimal implementation and various intermediate and final treatment outcomes. Largely using simple percentage adherence thresholds across the entire treatment period, suboptimal implementation has been associated with unsuccessful treatment outcomes in a variety of settings from Malawi to Israel, in both observational and randomised controlled trial datasets, and using a variety of methods to define and measure implementation [37, 45-50]. In observational datasets from Russia and the USA, this association extends to the development of drug resistance [51, 52], although in simulations it has not been consistently proven [53]. Recurrence of TB disease among pulmonary TB patients was higher with worse implementation in both the Yemen and Vietnam $[54,55]$.

Moving beyond adherence thresholds, in MDR-TB patients in Armenia and Abkhazia on DOT, BASTARD et al. [45] noted the criticality of gap length and the time between gaps. Odds of negative outcomes (treatment failure, death or default) nearly quadrupled with interruptions of three or more days and also short periods ( $<10$ days) between gaps. From a different angle in drug-sensitive pulmonary TB, a meta-regression by JoHNSTON et al. [56] found that treatment failure, acquired drug resistance and relapse were more common with thrice-weekly versus daily dosing. The IMPERIAL et al. [37] pooled meta-analysis looked at the impact of a 6 days in 7 versus a 7 days in 7 dosing strategy and found that the former increased the likelihood of an unfavourable outcome (broadly death, treatment failure, a lack of culture conversion, relapse, adverse events), as well as the implications of different adherence thresholds within this.

As for discontinuation, well-designed studies using DATs or other dose-by-dose monitoring systems will be essential to answer the remaining questions in this area. 


\section{What do different types of nonadherence mean for intervention and regimen design} and deployment?

Effectively preventing nonadherence to treatment not only requires interventions appropriately tailored to patients and healthcare systems, but also the type of nonadherence commonly displayed. Critically, the types of nonadherence displayed and their relationship to treatment outcomes may vary by population group, e.g. people living with HIV, individuals with other comorbidities, children and the elderly. Elucidating these relationships requires setting-by-setting data collection using tools such as DATs. This should include how variability in adherence throughout treatment determines the need for "step-up" interventions.

\section{Late or noninitiation}

Noninitiation of treatment after diagnosis can be due to a large number of complex factors, including the lack of accessibility of treatment, e.g. due to costs associated with attending the clinic; under-resourced or poorly functioning facilities; and stigma/lack of awareness of TB [57-59]. Here, interventions include broad systems-strengthening factors that will benefit the entire care cascade, such as better financing of healthcare systems; the provision of free TB drugs to everyone; and the removal of other financial barriers, e.g. through cash transfer programmes [60], as well as "pull factors" such as improvement in the quality of care; increasing awareness of/decreasing stigma around TB; and improving case detection/outreach. Factors such as strengthening the care cascade and reducing stigma may reduce late initiation, too.

\section{Discontinuation}

If discontinuation occurs early enough, even if it is relatively uncommon, it can form a large proportion of missed doses during treatment (figure 1c). As documented above, early discontinuation is also known to be highly detrimental to treatment outcomes. Therefore, settings should consider the relative burden of discontinuation versus other forms of nonadherence when planning for effective interventions to implement (table 1).

When it comes to intervention design, a single intervention may not address all discontinuation, as the drivers are not the same for every patient and sometimes reflect disengagement with care, rather than treatment.

One of the key implications for the development of shorter treatment regimens is their potential to reduce discontinuation [61], simply by reducing overall duration (table 1).

\section{Suboptimal implementation and interventions}

Intelligent intervention design should be influenced by the common form of suboptimal implementation (including long versus short gaps and erratic versus regular missed doses; figure 1d), their causes (i.e. treatment-related, individual knowledge and perceptions, social factors, systems issues, temporal factors and structural factors [12, 62-64]). Also influential is whether nonadherence is intentional or unintentional [11], however; making the distinction on an individual basis can be difficult and potentially fruitless.

To date, many interventions have sought to target individual-level cognitive or behavioural factors such as forgetfulness or "misconceptions" through SMS reminder systems, medication monitor box alarms, or the regular need to report for DOT or VOT [44]. More complex interventions are required to deal with multifactorial causes of nonadherence [7], such as rapid reporting and support systems. As TB tends to affect socially and economically deprived groups, interventions that focus on individual agency and behaviour, but do not account for social and structural barriers to care (as well as factors that influence a patient's ability to take medication regularly), are destined to work primarily for those who already have better capacity and social circumstances [65].

Critically, adherence to treatment is dynamic and can change in response to events and life circumstances of all kinds over time [66], producing ever-varying patterns of suboptimal implementation. Dose-by-dose monitoring systems that are accessible to healthcare services can be used to promote rapid responsiveness to the frequency and length of gaps that occur during treatment (table 1), as part of the partnership between patients and healthcare providers [67].

Polypharmacy is of substantial concern as a cause of nonadherence [68], and therefore population groups for whom this is an issue should have special consideration in intervention design.

\section{Suboptimal implementation and regimen forgiveness}

The "forgiveness" of treatment regimens reflects their ability to withstand unexpected gaps in dosing [69]. Forgiveness varies from drug to drug, depending on pharmacokinetic parameters, thus each drug will 
respond individually to different patterns of suboptimal implementation. The development of drug resistance is a key consideration; differing gap lengths can lead to divergent results. Within multidrug regimens, such as those used for $\mathrm{TB}$, the maintenance of sufficient drug blood levels to achieve an antibacterial effect depends upon the metabolism of all the component drugs and thus how they behave in combination. Dosing strategies may potentially be alterable to overcome nonforgiveness, but this should be undertaken in light of considerations surrounding the patient's medication-taking burden (e.g. the number of times doses need to be taken in a day) and whether or not combined pills containing different drugs of different characteristics are used [70, 71].

We provide two illustrations: not taking any treatment at a given time point versus not taking some of the drugs.

When all drugs are omitted at the same time, the implications of longer and shorter breaks should be considered separately. Longer gaps from treatment (4 days or more) can allow bacteria to restart replication. It is currently unknown how such an increase in the bacterial burden may affect treatment outcomes; it may prolong the treatment length required for a cure. Here, replication after previous exposure to antibiotics may facilitate the emergence of resistance.

Shorter breaks (1-2 days; table 1) may be a problem when different drugs within a combination regimen have different pharmacokinetic properties and therefore some may take a considerably longer time to clear and/or reach their therapeutic levels when the regimen is restarted. As a result, drug concentrations after the first dose and at steady state will differ considerably in some tissues or plasma. For example, STRYDOM et al. [72] have illustrated the effects of the slower accumulation of certain drugs in a pharmacokinetics study on TB patients undergoing lung resection surgery. In the most detailed study of its kind, the authors demonstrated that drug concentrations after the first dose of a drug differ from those at steady state - at least in some tissues - for ethambutol (shown in a different study [73]), pyrazinamide, moxifloxacin and linezolid. This was not the case for isoniazid, rifampicin and kanamycin. More studies of this type will help us understand how TB drugs accumulate and behave in relevant lesion types.

During instances when all drugs are omitted at the same time, the drug that clears more slowly will still be present after others, resulting in effective monotherapy during the gap. Even with perfect adherence, it is known that there are periods of effective monotherapy within each day [72]. The impact of such short bouts of monotherapy on the emergence of resistance is largely unknown. Drugs that require multiple days to reach their steady state levels may be below their therapeutic ranges for days after treatment resumes. Frequent short gaps may therefore keep levels below the therapeutic range for a longer period. Illustrations of how this would impact rifampicin and moxifloxacin levels in the lungs are presented (figure 3).

If FDCs are not used, it is also possible to suboptimally implement specific components of the regimen. During the continuation phase of treatment, suboptimal implementation of one drug will lead to monotherapy; the risk of drug resistance posed by monotherapy was illustrated by one of the first rifampicin trials in 1968 [77, 78]. As a result, the current ethical maximum for monotherapy studies is 14 days [79].

Further data in this area are required to better understand how gap lengths, timings and frequencies of suboptimal implementation carry the most risk for the emergence of resistance or in prolonging treatments, and how this is influenced by patient-by-patient variability in pharmacokinetics (e.g. isoniazid acetylator status) and clinical characteristics known to influence treatment success [37].

\section{The relationship between different types of nonadherence}

In addition to considering the different types of nonadherence in isolation, the relationships between them also have important implications for intervention design. For example, an approximate doubling in the likelihood of discontinuation in the presence of suboptimal implementation of $<80 \%$ versus $\geqslant 90 \%$ during the initiation phase of treatment has been demonstrated in data from China (table 1) [9, 12]. Early-stage dose-by-dose monitoring data from DATs could thus be highly valuable at indicating the patients who will later be in need of additional adherence support.

\section{Latent TB}

In our consideration of adherence to $\mathrm{TB}$ treatment up to this point, our focus has been on $\mathrm{TB}$ disease. Needless to say, the issues raised are equally important for latent tuberculosis infection (LTBI) and preventive treatment; there is still a need for a standardised taxonomic framework within which to discuss nonadherence. Unlike for drug-sensitive TB disease, adherence studies for LTBI need to take into account the different WHO-recommended regimen lengths and dosing patterns when applying this framework [80].

Numerous studies have documented how adherent patients are to LTBI treatment; such studies have a far greater focus on noninitiation than studies of treatment for TB disease, given the interest in 1) patients 


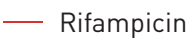

- Moxifloxacin
Only moxifloxacin above MIC (monotherapy)

Moxifloxacin below therapeutic ranges

No drugs above MIC (bacterial replication)
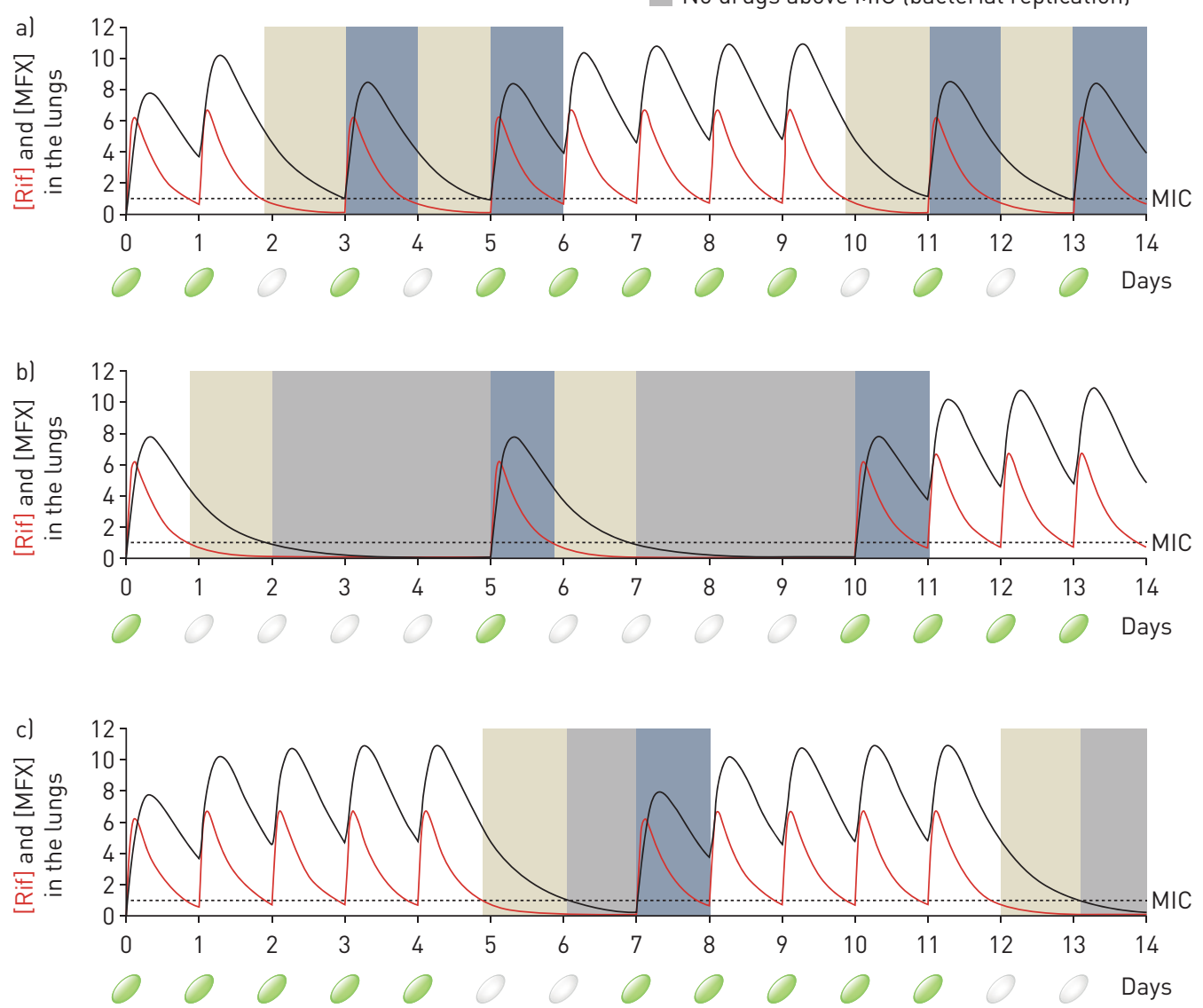

FIGURE 3 Different patterns in suboptimal implementation leads to divergent results. Rifampicin (red, 600 mg dose) and moxifloxacin (black, $400 \mathrm{mg}$ dose) concentrations were modelled in uninvolved lung tissues. This combination is currently being investigated in clinical trials [74], but the two drugs have very different pharmacokinetic properties. The three different plots show the same suboptimal implementation patterns as figure $1 \mathrm{~d}$. Patient 1 - short, irregular gaps. Patient 2 - long, irregular gaps. Patient 3 - regular gaps. The different shaded areas indicate different issues with drug concentrations. Cream indicates periods where only moxifloxacin is above the minimum inhibitory concentration (MIC). Above the MIC the drug either stops replication completely or eliminates bacteria, therefore during these periods there is an effective moxifloxacin monotherapy. Grey areas are periods where no drug is above the MIC; as a result, bacteria may eventually restart replication. Dark blue periods are when moxifloxacin concentrations do not reach the levels (therapeutic range) expected during proper adherence. In these cases, bacterial elimination rates for the given period may be lower than expected, therefore possibly delaying the time it takes to clear bacteria. The presented MIC cut-offs are mainly for illustration purposes, to indicate time periods where adverse events may occur due to differences in concentrations, rather than capturing events on a bacterial population level. Bacterial population dynamics are governed by multiple factors in addition to drug concentrations, e.g. the post-antibiotic effect. For instance, growth rates of bacteria may be affected by the post-antibiotic effect [75]. Furthermore, selection of resistance mechanisms also occurs at sub-MIC concentrations [76]. The plots were made with the model and parameters published by STRYDom et al. [72].

declining to take proffered treatment or 2) not being offered treatment [81, 82]. Additionally, the nature of LTBI makes treatment completion the marker of choice for treatment success by national TB programmes, thus the proportion of patients completing treatment has been extensively reviewed [81-84]. For both noninitiation and discontinuation, levels were highly variable between studies (7-99\% and 4-100\%, respectively). A global consensus as to which nonadherence patterns can be safely tolerated for LTBI regimens of different lengths is urgently needed. As with TB disease, this should also influence the design of interventions to promote adherence, as well as decisions on which regimens will be most effective in a given population group (balancing cost; the length of the regimen, its adverse event profile and the implications for adherence; and regimen efficacy).

\section{Conclusion}

As a global TB community, we find ourselves at a crossroads when it comes to treatment adherence. Through DATs, remote dose-by-dose treatment monitoring has become accessible like never before, and we have a great opportunity to deploy precision medicine approaches to develop and target adherence-promoting interventions. In the COVID-19 era, remote monitoring tools are all the more 


\section{Area}

\section{Global burden of different types of nonadherence}

\section{Trials versus normal treatment pathway Suboptimal implementation patterns}

\section{Relationship between the different components of adherence \\ Relationship between patterns and patient outcomes}

Regimen forgiveness

\section{Missing information}

A better determination of the distribution of nonadherence between late/noninitiation, suboptimal implementation and discontinuation.

Whether there are substantial differences between (and within) countries. Who displays each pattern.

Why different patterns are displayed.

The extent to which nonadherence varies between clinical trials and in normal care settings.

Improved estimates of the frequency and types of suboptimal implementation, explicitly excluding doses missed due to discontinuation.

Variability in patterns between settings and patients.

Causes of these patterns.

Whether early-stage indicators of nonadherence can predict later issues with nonadherence.

Specific mapping of how different nonadherence types and patterns impact treatment failure land the need to restart treatment) and the development of drug resistance, in order to prioritise cost-effective intervention development and roll-out.

The impact of the commonly displayed adherence patterns on forgiveness.

The implications of nonadherence to each drug within the multidrug regimen.

\section{Impact}

Stratification of settings/populations on the basis of the interventions that might be useful, including changes to healthcare processes and systems. Intelligent intervention design.

Aids decision-making surrounding the adoption of new regimens (operational efficacy).

Stratification of settings (e.g. by healthcare system)/ populations (e.g. by patient characteristics) on the basis of the interventions that might be useful.

Intelligent intervention design.

Inform clinicians as to which nonadherence patterns should trigger active intervention.

Stratification of settings/populations on the basis of the interventions that might be useful and when they should be "stepped up".

Intelligent intervention design.

Inform clinicians as to which nonadherence patterns should trigger active intervention.

Inform regimen design.

important for TB control due to the need to reduce patient contact with healthcare services (and we also note the likely impact of the disruption of the pandemic on adherence itself).

Important information is, however, missing. Further studies using tools such as DATs need to be rapidly undertaken to fill critical gaps in our knowledge where only limited data exist (table 2). It is essential that interventions are not adopted at the national scale without rigorous effectiveness and cost-effectiveness studies, such as that being undertaken by the ASCENT project across five countries [85]. During adoption, careful programmatic management is also required to avoid the wasteful parallel development of digital tools to report and manage DAT data [86].

Although we advocate in this paper for nonadherence to be considered as three separate issues, it is important to note that the underlying causes of each component may be similar and that each component may be interrelated. Effective interventions (such as those taking a stepped approach to enhanced treatment support, e.g. by more frequent contact with health systems or resolution of insecure housing, etc.), may work across several components of nonadherence, but this will not be known unless data are analysed in this fashion. Trials of different interventions should also seek to separate their impact on the different components of nonadherence [13].

The data that arise from studies such as those we propose will raise crucial questions for the future of TB control. For example, are levels of particularly problematic adherence issues low enough globally that it is not necessary to watch patients taking every single dose of their medication? Or should all patients be observed during the initiation phase, given the medication burden, higher replicating mycobacterial load, and connection between early suboptimal adherence and discontinuation, then allowed to self-medicate if no issues are observed? Can culturally adapted menus of interventions be developed to address the common forms of nonadherence for any given setting? Can we build predictive models to determine which patients are most likely to suffer from which problematic nonadherence issues?

To date, the TB literature has largely treated all missed doses of treatment as equally problematic and equally common. By harnessing the power of dose-by-dose adherence data, particularly through DATs, we can determine which patterns of missingness are "more equal than others", a finding that could revolutionise our approach to nonadherence. 
Author contributions: All authors contributed to the conception of the work. M. Flook, H.R. Stagg, A. Martinecz and P. Abel zur Wiesch contributed to the acquisition, analysis and interpretation of data/literature for the work. All authors drafted the work/revised it critically for important intellectual content. All authors give final approval of the manuscript version to be published and agree to be accountable for all aspects of the work in ensuring that questions related to the accuracy or integrity of any part of the work are appropriately investigated and resolved.

Conflict of interest: H.R. Stagg reports that she is the Chief Investigator on, and supported by, Medical Research Council MR/R008345/1; and a co-applicant on NIHR grant 16/88/06 (the IMPACT study), which contains a small salary contribution. She also reports travel and subsistence support from events organised by the Korean CDC and the Latvian Society Against Tuberculosis, some of the sponsorship for which was obtained from Otsuka and Johnson and Johnson. M. Flook reports grants from the Medical Research Council, UK, during the conduct of the study. A. Martinecz has nothing to disclose. K. Kielmann has nothing to disclose. P. Abel zur Wiesch has nothing to disclose. A.S. Karat reports grants awarded to the London School of Hygiene \& Tropical Medicine (LSHTM) from the World Health Organization and the Medical Research Council, UK; grants awarded to University College London (subcontract to Queen Mary University) from the National Institute of Health Research, UK; grants awarded to the LSHTM from the Economic and Social Research Council, UK, and The Bloomsbury SET (Research England); grants awarded to Imperial College London from The Colt Foundation, UK; grants awarded to the LSHTM from Viiv Healthcare, USA; consultancy fees from The Aurum Institute, South Africa, Edanz Group, Japan, and Pastest, UK; an external marker fee from The University of Cape Town, South Africa; travel and subsistence support from Kyoto University, Japan, Vital Strategies, Singapore, and Bloomberg Philanthropies, USA; and costs of open access publishing from the Bill \& Melinda Gates Foundation, USA, all outside the submitted work. M.C.I. Lipman reports grants from National Institute for Health Research, UK, during the conduct of the study. D.J. Sloan has nothing to disclose. E.F. Walker has nothing to disclose. K.L. Fielding has nothing to disclose.

Support statement: H.R. Stagg and M. Flook are supported by the Medical Research Council (grant number MR/ R008345/1). H.R. Stagg, A.S. Karat and M.C.I. Lipman are supported by the National Institute for Health Research (NIHR) Health Technology Assessment Programme, UK (grant number 16/88/06). The views expressed are those of the author(s) and not necessarily those of the UK's National Health Service, the NIHR or the Department of Health and Social Care. The funders did not play a role in the writing of the manuscript or the decision to submit for publication. None of the authors have been paid to write this article by a pharmaceutical company or other agency. The corresponding author had full access to all the data in the study and has final responsibility for the decision to submit for publication. Funding information for this article has been deposited with the Crossref Funder Registry.

\section{References}

$1 \quad$ Medical Research Council. Streptomycin treatment of pulmonary tuberculosis. Br Med J 1948; 2: 769-782.

2 World Health Organization. Treatment of tuberculosis: guidelines. 4th Edn. Geneva, World Health Organization, 2010. Available from: www.who.int/tb/publications/2010/9789241547833/en/. Date last accessed: July 1, 2013.

3 Fox W. The problem of self-administration of drugs; with particular reference to pulmonary tuberculosis. Tubercle 1958; 39: 269-274.

4 Obermeyer Z, Abbott-Klafter J, Murray CJ. Has the DOTS strategy improved case finding or treatment success? An empirical assessment. PLoS One 2008; 3: e1721.

5 Subbaraman R, de Mondesert L, Musiimenta A, et al. Digital adherence technologies for the management of tuberculosis therapy: mapping the landscape and research priorities. BMJ Glob Health 2018; 3: e001018.

6 Nsengiyumva NP, Mappin-Kasirer B, Oxlade O, et al. Evaluating the potential costs and impact of digital health technologies for tuberculosis treatment support. Eur Respir J 2018; 52: 1801363.

7 Stagg HR, Abubakar I, Campbell CN, et al. IMPACT study on intervening with a manualised package to achieve treatment adherence in people with tuberculosis: protocol paper for a mixed-methods study, including a pilot randomised controlled trial. BMJ Open 2019; 9: e032760.

8 Lewis JJ, Liu X, Zhang Z, et al. Evaluation of a medication monitor-based treatment strategy for drug-sensitive tuberculosis patients in China: study protocol for a cluster randomised controlled trial. Trials 2018; 19: 398.

9 Liu X, Lewis JJ, Zhang $\mathrm{H}$, et al. Effectiveness of electronic reminders to improve medication adherence in tuberculosis patients: a cluster-randomised trial. PLoS Med 2015; 12: e1001876.

10 Story A, Aldridge RW, Smith CM, et al. Smartphone-enabled video-observed versus directly observed treatment for tuberculosis: a multicentre, analyst-blinded, randomised, controlled superiority trial. Lancet 2019; 393: $1216-1224$.

11 Horne R, Weinman J, Barber N, et al. Concordance, adherence and compliance in medicine taking. Southampton (UK), National Co-ordinating Centre for NHS Service Delivery and Organisation R \& D (NCCSDO), 2005. www. netscc.ac.uk/hsdr/files/project/SDO_FR_08-1412-076_V01.pdf. Date last accessed October 21, 2016.

12 Stagg HR, Lewis JJ, Liu X, et al. Temporal factors and missed doses of tuberculosis treatment. A causal associations approach to analyses of digital adherence data. Ann Am Thorac Soc 2020; 17: 438-449.

13 Vernon A, Fielding K, Savic R, et al. The importance of adherence in tuberculosis treatment clinical trials and its relevance in explanatory and pragmatic trials. PLoS Med 2019; 16: e1002884.

14 Vrijens B, De GS, Hughes DA, et al. A new taxonomy for describing and defining adherence to medications. $\mathrm{Br} \mathrm{J}$ Clin Pharmacol 2012; 73: 691-705.

15 World Health Organization. Guidelines for treatment of drug-susceptible tuberculosis and patient care (2017 update). Geneva, World Health Organization, 2010. Available from: www.who.int/tb/publications/2017/dstb_ guidance_2017/en/. Date last accessed: August 5, 2017.

16 World Health Organization. WHO consolidated guidelines on drug resistant tuberculosis treatment. Geneva, World Health Organization, 2019. Available from: www.who.int/tb/publications/2019/consolidated-guidelinesdrug-resistant-TB-treatment/en/. Date last accessed: February 7, 2020.

17 World Health Organization. Definitions and reporting framework for tuberculosis (2013 revision). Geneva, World Health Organization, 2014. Available from: www.who.int/tb/publications/definitions/en/. Date last accessed: October 25, 2016. 
18 MacPherson P, Houben RM, Glynn JR, et al. Pre-treatment loss to follow-up in tuberculosis patients in low- and lower-middle-income countries and high-burden countries: a systematic review and meta-analysis. Bull World Health Organ 2014; 92: 126-138.

19 Naidoo P, Theron G, Rangaka MX, et al. The South African tuberculosis care cascade: estimated losses and methodological challenges. J Infect Dis 2017; 216: Suppl. 7, S702-S713.

20 Arinaminpathy N, Chin DP, Sachdeva KS, et al. Modelling the potential impact of adherence technologies on tuberculosis in India. Int J Tuberc Lung Dis 2020; 24: 526-533.

21 World Health Organization, Stop TB Partnership, The Global Fund to Fight AIDS TB and Malaria. Reach the 3 million: Find. Treat. Cure TB. Date last accessed: 7 April, 2020. https://www.who.int/campaigns/tb-day/2014/ campaign-brochure/en/.

22 World Health Organization. Global Tuberculosis Report 2019. Geneva, World Health Organization, 2019. Available from: www.who.int/tb/publications/global_report/en/. Date last accessed: November 5, 2019.

23 Boyd R, Ford N, Padgen P, et al. Time to treatment for rifampicin-resistant tuberculosis: systematic review and meta-analysis. Int J Tuberc Lung Dis 2017; 21: 1173-1180.

24 Sreeramareddy CT, Qin ZZ, Satyanarayana S, et al. Delays in diagnosis and treatment of pulmonary tuberculosis in India: a systematic review. Int J Tuberc Lung Dis 2014; 18: 255-266.

25 World Health Organization. Diagnostic and treatment delay in tuberculosis. Geneva, World Health Organization, 2006. Available from: https://apps.who.int/iris/handle/10665/116501. Date last accessed: February 11, 2020.

26 Chen Y, Yuan Z, Shen X, et al. Time to multidrug-resistant tuberculosis treatment initiation in association with treatment outcomes in Shanghai, China. Antimicrob Agents Chemother 2018; 62: e02259-17.

27 Melsew YA, Doan TN, Gambhir M, et al. Risk factors for infectiousness of patients with tuberculosis: a systematic review and meta-analysis. Epidemiol Infect 2018; 146: 345-353.

28 Asres A, Jerene D, Deressa W. Delays to treatment initiation is associated with tuberculosis treatment outcomes among patients on directly observed treatment short course in Southwest Ethiopia: a follow-up study. BMC Pulm Med 2018; 18: 64

29 Htun YM, Khaing TMM, Aung NM, et al. Delay in treatment initiation and treatment outcomes among adult patients with multidrug-resistant tuberculosis at Yangon Regional Tuberculosis Centre, Myanmar: a retrospective study. PLoS One 2018; 13: e0209932.

30 Walker IF, Shi O, Hicks JP, et al. Analysis of loss to follow-up in 4099 multidrug-resistant pulmonary tuberculosis patients. Eur Respir J 2019; 54: 1800353

31 Kruk ME, Schwalbe NR, Aguiar CA. Timing of default from tuberculosis treatment: a systematic review. Trop Med Int Health 2008; 13: 703-712.

32 Iseman MD. Tuberculosis therapy: past, present and future. Eur Respir I Suppl 2002; 36: 87s-94s.

33 Long-term follow-up of a clinical trial of six-month and four-month regimens of chemotherapy in the treatment of pulmonary tuberculosis. Singapore Tuberculosis Service/British Medical Research Council. Am Rev Respir Dis 1986; 133: 779-783

34 Gillespie SH, Crook AM, McHugh TD, et al. Four-month moxifloxacin-based regimens for drug-sensitive tuberculosis. N Engl J Med 2014; 371: 1577-1587.

35 Jindani A, Harrison TS, Nunn AJ, et al. High-dose rifapentine with moxifloxacin for pulmonary tuberculosis. N Engl J Med 2014; 371: 1599-1608.

36 Merle CS, Fielding K, Sow OB, et al. A four-month gatifloxacin-containing regimen for treating tuberculosis. N Engl J Med 2014; 371: 1588-1598.

37 Imperial MZ, Nahid P, Phillips PPJ, et al. A patient-level pooled analysis of treatment-shortening regimens for drug-susceptible pulmonary tuberculosis. Nat Med 2018; 24: 1708-1715.

38 Fagundez G, Perez-Freixo H, Eyene J, et al. Treatment adherence of tuberculosis patients attending two reference units in Equatorial Guinea. PLoS One 2016; 11: e0161995.

39 Gube AA, Debalkie M, Seid K, et al. Assessment of anti-TB drug nonadherence and associated factors among TB patients attending TB clinics in Arba Minch governmental health institutions, Southern Ethiopia. Tuberc Res Treat Print 2018; 2018: 3705812 .

40 Alegria-Flores $\mathrm{K}$, Weiner BJ, Wiesen CA, et al. Innovative approach to the design and evaluation of treatment adherence interventions for drug-resistant TB. Int J Tuberc Lung Dis 2017; 21: 1160-1166.

41 AlSahafi AJ, Shah HBU, AlSayali MM, et al. High non-compliance rate with anti-tuberculosis treatment: a need to shift facility-based directly observed therapy short course (DOTS) to community mobile outreach team supervision in Saudi Arabia. BMC Public Health 2019; 19: 1168.

42 Cai EZ, Chua SM, Tan M, et al. Tuberculosis care: enhancing directly observed therapy in a peri-urban, low socioeconomic status neighbourhood. Singapore Med J 2019; 60: 334-336.

43 Zegeye A, Dessie G, Wagnew F, et al. Prevalence and determinants of anti-tuberculosis treatment non-adherence in Ethiopia: a systematic review and meta-analysis. PLoS One 2019; 14: e0210422.

44 World Health Organization. Handbook for the use of digital technologies to support tuberculosis medication adherence. Geneva, World Health Organization, 2018. Available from: www.who.int/tb/publications/2018/TB_ medication_adherence_handbook_2018/en/. Date last accessed: November 1, 2018.

45 Bastard M, Sanchez-Padilla E, Hewison C, et al. Effects of treatment interruption patterns on treatment success among patients with multidrug-resistant tuberculosis in Armenia and Abkhazia. J Infect Dis 2015; 211: 1607-1615.

46 Chirwa T, Nyasulu P, Chirwa E, et al. Levels of tuberculosis treatment adherence among sputum smear positive pulmonary tuberculosis patients attending care at Zomba Central Hospital, southern Malawi, 2007-2008. PLoS One 2013; 8: e63050.

47 Kayigamba FR, Bakker MI, Mugisha V, et al. Adherence to tuberculosis treatment, sputum smear conversion and mortality: a retrospective cohort study in 48 Rwandan clinics. PLoS One 2013; 8: e73501.

48 Thomas A, Gopi PG, Santha T, et al. Predictors of relapse among pulmonary tuberculosis patients treated in a DOTS programme in South India. Int J Tuberc Lung Dis 2005; 9: 556-561.

49 Zhdanov V, Bilenko N, Mor Z. Risk factors for recurrent tuberculosis among successfully treated patients in Israel, 1999-2011. Isr Med Assoc J 2017; 19: 237-241.

50 Tola HH, Holakouie-Naieni K, Mansournia MA, et al. Intermittent treatment interruption and its effect on multidrug resistant tuberculosis treatment outcome in Ethiopia. Sci Rep 2019; 9: 20030. 
51 Bradford WZ, Martin JN, Reingold AL, et al. The changing epidemiology of acquired drug-resistant tuberculosis in San Francisco, USA. Lancet 1996; 348: 928-931.

52 Shin SS, Keshavjee S, Gelmanova IY, et al. Development of extensively drug-resistant tuberculosis during multidrug-resistant tuberculosis treatment. Am J Respir Crit Care Med 2010; 182: 426-432.

53 Srivastava S, Pasipanodya JG, Meek C, et al. Multidrug-resistant tuberculosis not due to noncompliance but to between-patient pharmacokinetic variability. J Infect Dis 2011; 204: 1951-1959.

54 Anaam MS, Alrasheedy AA, Alsahali S, et al. Rate and risk factors of recurrent tuberculosis in Yemen: a 5-year prospective study. Infect Dis (Lond) 2020; 52: 161-169.

55 Bestrashniy J, Nguyen VN, Nguyen TL, et al. Recurrence of tuberculosis among patients following treatment completion in eight provinces of Vietnam: a nested case-control study. Int J Infect Dis 2018; 74: 31-37.

56 Johnston JC, Campbell JR, Menzies D. Effect of intermittency on treatment outcomes in pulmonary tuberculosis an updated systematic review and metaanalysis. Clin Infect Dis 2017; 64: 1211-1220.

57 Skinner D, Claassens M. It's complicated: why do tuberculosis patients not initiate or stay adherent to treatment? A qualitative study from South Africa. BMC Infect Dis 2016; 16: 712.

58 Kelkar-Khambete A, Kielmann K, Pawar S, et al. India's Revised National Tuberculosis Control Programme: looking beyond detection and cure. Int J Tuberc Lung Dis 2008; 12: 87-92.

59 Pradhan A, Kielmann K, Gupte H, et al. What 'outliers' tell us about missed opportunities for tuberculosis control: a cross-sectional study of patients in Mumbai, India. BMC Public Health 2010; 10: 263.

60 Reis-Santos B, Shete P, Bertolde A, et al. Tuberculosis in Brazil and cash transfer programs: a longitudinal database study of the effect of cash transfer on cure rates. PLoS One 2019; 14: e0212617.

61 Grace AG, Mittal A, Jain S, et al. Shortened treatment regimens versus the standard regimen for drug-sensitive pulmonary tuberculosis. Cochrane Database Syst Rev 2019; 12: CD012918.

62 World Health Organization. Adherence to long-term therapies: Evidence for action. Geneva: Switzerland, 2003 https://www.who.int/chp/knowledge/publications/adherence_report/en/.

63 Munro SA, Lewin SA, Smith HJ, et al. Patient adherence to tuberculosis treatment: a systematic review of qualitative research. PLoS Med 2007; 4: e238.

64 Amico KR, Mugavero M, Krousel-Wood MA, et al. Advantages to using social-behavioral models of medication adherence in research and practice. J Gen Intern Med 2018; 33: 207-215.

65 Saunders MJ, Wingfield T, Tovar MA, et al. Mobile phone interventions for tuberculosis should ensure access to mobile phones to enhance equity - a prospective, observational cohort study in Peruvian shantytowns. Trop Med Int Health 2018; 23: 850-859.

66 Kielmann K, Vidal N, Riekstina V, et al. "Treatment is of primary importance, and social assistance is secondary": a qualitative study on the organisation of tuberculosis (TB) care and patients' experience of starting and staying on TB treatment in Riga, Latvia. PLoS One 2018; 13: e0203937.

67 Thiam S, LeFevre AM, Hane F, et al. Effectiveness of a strategy to improve adherence to tuberculosis treatment in a resource-poor setting: a cluster randomized controlled trial. JAMA 2007; 297: 380-386.

68 Jeon CY, Murray MB. Diabetes mellitus increases the risk of active tuberculosis: a systematic review of 13 observational studies. PLoS Med 2008; 5: e152.

69 Urquhart J. The electronic medication event monitor. Lessons for pharmacotherapy. Clin Pharmacokinet 1997; 32 345-356.

70 Weiner M, Benator D, Burman W, et al. Association between acquired rifamycin resistance and the pharmacokinetics of rifabutin and isoniazid among patients with HIV and tuberculosis. Clin Infect Dis 2005; 40: 1481-1491.

71 Weiner M, Burman W, Vernon A, et al. Low isoniazid concentrations and outcome of tuberculosis treatment with once-weekly isoniazid and rifapentine. Am J Respir Crit Care Med 2003; 167: 1341-1347.

72 Strydom N, Gupta SV, Fox WS, et al. Tuberculosis drugs' distribution and emergence of resistance in patient's lung lesions: a mechanistic model and tool for regimen and dose optimization. PLoS Med 2019; 16: e1002773.

73 Zimmerman M, Lestner J, Prideaux B, et al. Ethambutol partitioning in tuberculous pulmonary lesions explains its clinical efficacy. Antimicrob Agents Chemother 2017; 61: e00924-17.

74 Boeree MJ, Heinrich N, Aarnoutse R, et al. High-dose rifampicin, moxifloxacin, and SQ109 for treating tuberculosis: a multi-arm, multi-stage randomised controlled trial. Lancet Infect Dis 2017; 17: 39-49.

75 Chan CY, Au-Yeang C, Yew WW, et al. In vitro postantibiotic effects of rifapentine, isoniazid, and moxifloxacin against Mycobacterium tuberculosis. Antimicrob Agents Chemother 2004; 48: 340-343.

76 Sandegren L. Selection of antibiotic resistance at very low antibiotic concentrations. Ups J Med Sci 2014; 119: 103-107.

77 Baronti A, Lukinovich N. A pilot trial of rifampicin in tuberculosis. Tubercle 1968; 49: 180-186.

78 Grobbelaar M, Louw GE, Sampson SL, et al. Evolution of rifampicin treatment for tuberculosis. Infect Genet Evol 2019; 74: 103937.

79 Davies G, Boeree M, Hermann D, et al. Accelerating the transition of new tuberculosis drug combinations from Phase II to Phase III trials: new technologies and innovative designs. PLoS Med 2019; 16: e1002851.

80 World Health Organization. Latent TB Infection: Updated and consolidated guidelines for programmatic management. Geneva, World Health Organization, 2018. Available from: www.who.int/tb/publications/2018/ latent-tuberculosis-infection/en/. Date last accessed: July 15, 2020.

81 Liu Y, Birch S, Newbold KB, et al. Barriers to treatment adherence for individuals with latent tuberculosis infection: a systematic search and narrative synthesis of the literature. Int J Health Plann Manage 2018; 33: e416-ee33.

82 Sandgren A, Vonk Noordegraaf-Schouten M, van Kessel F, et al. Initiation and completion rates for latent tuberculosis infection treatment: a systematic review. BMC Infect Dis 2016; 16: 204.

83 Assefa Y, Assefa Y, Woldeyohannes S, et al. 3-month daily rifampicin and isoniazid compared to 6- or 9-month isoniazid for treating latent tuberculosis infection in children and adolescents less than 15 years of age: an updated systematic review. Eur Respir J 2018; 52: 1800395.

84 Hirsch-Moverman Y, Daftary A, Franks J, et al. Adherence to treatment for latent tuberculosis infection systematic review of studies in the US and Canada. Int J Tuberc Lung Dis 2008; 12: 1235-1254.

85 ASCENT (Adherence Support Coalition to End TB). Empowering Patients through Digital Adherence Technology. Available from: www.digitaladherence.org/. Date last accessed: July 16, 2020.

86 Falzon D, Timimi H, Kurosinski P, et al. Digital health for the End TB Strategy: developing priority products and making them work. Eur Respir J 2016; 48: 29-45. 